\title{
Los ecosistemas de manglar frente al cambio climático global
}

\author{
Alejandro Yáñez-Arancibia \\ Robert R. Twilley ${ }^{2}$ \\ Ana Laura Lara-Domínguez ${ }^{3}$
}

\section{RESUMEN}

Los manglares constituyen un importante recurso forestal en toda la banda intertropical del planeta. Las acciones antrópicas acrecientan los riesgos sobre los ecosistemas y esto ha sido más catastrófico que el propio cambio climático global. Este último induce nuevas incertidumbres en la estabilidad ambiental y aumenta la vulnerabilidad de los hábitats críticos. Frente al desafío que enfrentan los sistemas económicos, sociales y ecológicos se presentan evidencias de estructura funcional de este sistema ecológico, planteando la hipótesis que "los manglares como hábitat forestado crítico de la zona costera presentan respuestas de acomodación frente a la variabilidad ambiental que induce el cambio global, desarrollando un papel estructural y funcional clave en la estabilidad de la línea de costa, la persistencia de hábitats y biodiversidad, el metabolismo del ecosistema, reduciendo riesgos e incertidumbre para el desarrollo sustentable del uso de sus recursos".

\section{PALABRAS CLAVE:}

Recurso forestal, manglares, humedales intermareales tropicales, estructura uncional del ecosistema costero, cambio climático global.

\section{ABSTRACT}

The mangroves constitute an important forest resource in all of the intertropical band of the planet. Mankind actions increase the threat and risks over the ecosystems and this has been more catastrophic than the global climate change itself. This induces new uncertainties in the environmental stability and augments the vulnerability of the critical habitats. Considering the challenge that the economic, social and ecological systems face -in order to know and mitigate the global climate change-, evidences of the functional structure of this ecological system are presented, posing the hypothesis the "the mangroves as a critical forest habitat of the coastal zone present accomodation responses to the environmental variability that induces global change playing an structural and functional role in the stability of the coastline, the persistance of habitats and biodiversity, the metabolism of the ecosystem, reducing risks and uncertainty for the sustainable development of the use of its resources".

KEY WORDS:

Forest resource, mangroves, tropical intertidal wetlands, functional structure of the coastal ecosystems, global climatic change.

1 Programa Recursos Costeros. Instituto de Ecología A.C. Apdo. Postal 63. Xalapa, Ver. 91000 México. aya@ecologia.edu.mx

2 Department of Biology. University of Southwestern Louisiana. P.O. Box 42451. Lafayette 70504. Louisiana EUA.rtwilley@usl.edu

3 Coastal Ecology Institute. Graduate Program Department of Oceanography and Coastal Sciences. Louisiana State University. Baton Rouge 70803. Louisiana EUA y Centro Epomex-UAC, Apdo. Postal 520, Campeche, Camp.24030 México. anallara@epomex.uacam.mx

Manuscrito recibido para su publicación el 6 de octubre de 1998 


\section{INTRODUCCION}

Los manglares constituyen un importante recurso forestal en toda la banda intertropical del planeta (ca. $240 \mathrm{x}$ $10^{3} \mathrm{~km}^{2}$, pero también se han señalado 15 $x 10^{6}$ ha). Son los árboles que sostienen la biodiversidad de los ecosistemas costeros tropicales, en los humedales forestados intermareales y áreas de influencia tierra adentro. En México, la SARH indicó en el Inventario Forestal Nacional de los años 70 's una superficie de $1.5 \times 10^{6}$ ha ; pero el Inventario Forestal de Gran Visión de 1992 señaló que sólo quedan en existencia poco más de $500 \times 10^{3}$ ha, reflejando una tasa de deforestación de $60 \%$ en esos 20 años. Para fines de la década de los 90's la tasa de deforestación de manglares en México habrá llegado al $70 \%$ o más. Las acciones antrópicas acrecientan las amenazas y riesgos naturales sobre los ecosistemas y esto ha sido más catastrófico que el propio cambio climático global.

La ubicación de estos humedales forestados en la interfase tierra-mar (formando conjuntos inter-dependientes) ligan el ambiente marino-costero con el paisaje terrestre-costero. Aún cuando los manglares predominan en zonas de mayor influencia de sedimentos terrígenos (p.e. deltas fluviales, lagunas costeras y estuarios), también pueden ser encontrados en la línea de costa de plataformas carbonatadas, con limitada o nula influencia de drenaje dulce-acuícola superficial. Esta heterogeneidad de hábitats y contrastes hidrológicos, fisiográficos y geomorfológicos, da como resultado diversos ecosistemas de manglar, cada uno con características específicas de estructura y función (p.e. ribereño, de borde, de cuenca, de hamacas, o enanos). Aunque existen relativamente pocas especies de árboles costeros en ecosistemas de manglar (p.e. 54 especies), los componentes de biodiversidad de estos ecosistemas son únicos debido a que incluyen nichos estructurales, son fronteras a nivel de eco-regiones, y actúan como refugio para numerosa fauna y especies microbianas. Estas especies halofíticas se reúnen en 8 familias que contienen mangles verdaderos y 12 géneros: Avicenniaceae (Avicennia), Chenopodiaceae (Suaeda), Combretaceae (Laguncularia, Lumnitzera), Meliaceae (Conocarpus, Xylocarpus), Myrsinaceae (Aegiceras), Plumbagina-ceae (Aegialitis), Rhizophoraceae (Rhizophora, Bruguiera), Sonneratiaceae (Sonneratia); incluyendo un total de 11 especies en el nuevo mundo y 36 en el Indo Pacífico y Africa, estando representadas en México 4 especies: Rhizophora mangle, Avicennia nitida, Laguncularia racemosa y Conocarpus erectus.

El paisaje costero donde se desarrollan los manglares, es vulnerable por fuerzas naturales episódicas de alto impacto, p.e. huracanes, deslizamientos de tierras, subsidencia, diapirismo de lodo, aumento acelerado del nivel medio del mar, y el cambio climático global. En el umbral del siglo XXI, los sistemas económicos, sociales y ecológicos del Golfo de México y Caribe, tienen un gran desafío en intensificar esfuerzos dirigidos al conocimiento y a la mitigación del cambio climático global, donde una gran limitante es todavía la poca comprensión de la estructura y el funcionamiento de los ecosistemas costeros.

El fenómeno de "El Niño" combinado con los efectos que induce la ruptura de la capa de ozono atmosférica, y el efecto invernadero sobre el planeta, están afectando los patrones de temperatura, precipitación pluvial, depresiones tropicales, huracanes, descarga de ríos, y variación del nivel medio del mar, induciendo nuevas incertidumbres en la 
estabilidad ambiental de los hábitats críticos. Los manglares no son la excepción, pero muestran sutiles evidencias para contender con mejor éxito que otros humedales costeros frente a esta nueva variabilidad físico ambiental de ritmo acelerado.

México es uno de los países comprometidos con este desafío, lo cual ha sido claramente expresado en el documento publicado por la SEMARNAP (1997), denominado "México Primera Comunicación Nacional ante la Convención Marco de las Naciones Unidas sobre el Cambio Climático". Aquí se destaca de manera puntual a la zona costera como el escenario base para la comprensión de la problemática del cambio global y la vulnerabilidad de los ecosistemas. Humedales costeros, cuencas bajas de los ríos, sistemas deltáicos, lagunas costeras, y la línea de costa, muestran pérdida de hábitats críticos por subsidencia, variación del nivel medio del mar, y erosión litoral, como efecto al cambio climático global particularmente evidente en el Golfo de México. Esto induce inestabilidad ecológica y agrega un elemento de riesgo en los proyectos de desarrollo económico y social en las costas.

La hipótesis principal planteada en este análisis indica que estos ecosistemas de humedales forestados tienen paradójicamente una gran capacidad de acomodación al mismo tiempo de su alta fragilidad como hábitat crítico, lo cual les permite contender con éxito frente a la variabilidad ambiental de periodo-corto que está induciendo el cambio climático global.

\section{ESTRUCTURA, BIODIVERSIDAD FUNCIONAL Y VALORACION DEL ECOSISTEMA}

Los efectos producidos por el cambio climático global, aparentemente están siendo evidentes a mayor celeridad que lo estimado hace veinticinco años. Es decir, en periodos cortos o más aún en tiempo real, el hombre está observando colapsos ecológicos y su efecto en los sistemas sociales y económicos como consecuencia que se atribuye al cambio climático global. La intensidad específica del fenómeno "El Niño" en los periodos 1982-1983 y 19971998 son las principales alarmas con que está finalizando este siglo. Si se intenta analizar la capacidad de los ecosistemas de manglar para responder a los efectos del cambio global y visualizar medidas de mitigación, es preciso comprender la dinámica del ecosistema (es decir, su estructura funcional), su biodiversidad funcional (o sea, el papel ecológico de sus componentes estructurales conspicuos), así como también estimar económicamente las funciones o servicios del ecosistema (la valuación de las funciones ecológicas).

\section{Estructura funcional}

El mosaico de hábitats de manglares provee gran variedad de componentes de biodiversidad que son importantes para la función y calidad ambiental de los ecosistemas estuarinos tropicales. La función ecológica dominante de los manglares es el mantenimiento de hábitats costero-marinos y la provisión concomitante de alimento y refugio para una gran variedad de organismos a diferentes niveles tróficos. Además los manglares juegan un papel principal en mantener la calidad del agua y la estabilidad de la línea de costa, controlando la distribución de nutrientes y sedimentos en aguas estuarinas.

Estos pantanos forestados son únicos donde las mareas modulan el intercambio de agua, nutrientes, sedimentos y organismos entre ecosistemas costeros intermareales tropicales. También los ríos y sus cuencas bajas vinculan la descarga de sedimentos y nutrientes desde el continente, modulando la productividad y biogeoquímica de estuarios tropicales, acoplándose esta dinámica con ecosistemas vecinos. Las múltiples funciones de los manglares inducen una productividad primaria y producción secundaria extremadamente alta en costas tropicales. 
Los manglares se presentan dentro de cinco grupos básicos de ambientes costeros, dependiendo de una combinación de energías geológicas, incluyendo la influencia relativa de la precipitación pluvial, descarga de ríos, amplitud de mareas, turbidez y fuerza del oleaje (manglares de cuenca, manglar ribereño, manglar de borde, manglar de islotes o hamacas, manglar enano), esencialmente en litorales con influencia de sedimentos terrígenos (deltas fluviales, lagunas deltáicas, lagunas costeras, estuarios). La distribución espacial de estos tipos ecológicos dentro de la zona costera puede ser ilustrada en el gradiente propuesto por Gilmore y Snedaker incluido en la figura 1. Los dos tipos de clasificaciones de los ecosistemas de manglar, el geológico y el ecológico, representan diferentes niveles de organización del paisaje costero y en conjunto pueden ser usados para integrar diferentes escalas de factores ambientales que controlan los atributos de la estructura forestal de los manglares (Fig. 2).

Las hojas producidas en el follaje del manglar, influyen en el ciclo de nutrientes inorgánicos en el piso del bosque, y la exportación de materia orgánica hacia las aguas costeras oceánicas (Fig.3). Por lo tanto, la dinámica de la foliación del manglar, incluyendo productividad, descomposición y exportación, ejerce influencia los presupuestos de nutrientes y materia orgánica en el ecosistema. Los manglares son ecosistemas forestados y muchas de las funciones ecológicas de ciclos de nutrientes descritas para bosques terrestres, pueden ocurrir también en estos bosques de humedales intermareales. Así, el ciclo del nitrógeno en el follaje del bosque está acoplado con la dinámica de los nutrientes en los suelos del mismo, y estos están influidos por la ecología nutricional que es especie-específico en estos árboles.

La acumulación de hojarasca sobre el piso del manglar puede ser un importante factor para la inmovilización de nutrientes durante la descomposición. La concentración del nitrógeno en la hojarasca, generalmente se incrementa durante la descomposición sobre el piso del bosque, aparentemente siendo una función de la demanda de este sustrato por la microbiota que coloniza el detritus. En bosques de Rhizophora la tasa de descomposición de hojarasca es más baja y la inmovilización de nitrógeno es más alta, que en bosques de Avicennia, como resultado de una alta tasa C:N. Sin embargo, hay evidencias que la retranslocación de nutrientes previo a que ocurra la defoliación, es mayor en Rhizophora que en Avicennia, contribuyendo esto a la alta tasa $\mathrm{C}: \mathrm{N}$ en hojarasca de Rhizophora. Esto sugiere que más nitrógeno puede ser reciclado en el follaje de los bosques dominados por Rhizophora que en Avicennia. Complementariamente, la mayor remineralización de nitrógeno en la hojarasca de Avicennia puede suplir la alta demanda de nitrógeno en el follaje de este género. Estas ideas sugieren que la dominancia alterna de ambos géneros de acuerdo a gradientes de zonación, balancea la dinámica del nitrógeno entre el follaje y el suelo del ecosistema de manglar, con la ventaja de ser un mecanismo flexible frente al cambio climático global y su efecto en los litorales tropicales.

La productividad de los manglares, tanto primaria como secundaria, generalmente se asocia con el concepto de "exportación" en ecosistemas lagunarestuarinos. Una razón de esto puede ser la característica de los manglares de ubicarse en un ambiente modulado por la amplitud de las mareas y el flujo de los ríos. Por otra parte, la productividad de estos bosques se relaciona con la fisiografía y el origen geológico del paisaje donde se sitúan, así como de sus respectivas características hidrológicas. Esta conclusión se basa principalmente sobre el intercambio de materia orgánica en los manglares, aunque hay evidencias que el reciclamiento de 
nutrientes puede también variar a lo largo de un continuo en hidrología. El tiempo de residencia de la hojarasca en el piso del bosque está fuertemente controlada por la frecuencia del flujo de la marea y el volumen de descarga de agua dulce. A nivel global, el promedio de exportación de carbón desde los manglares es ca. 210 $\mathrm{gCm}^{-2}$ año ${ }^{-1}$, con un rango que varía entre 1.86 a $420 \mathrm{gCm}^{-2}$ año $^{-1}$, y ca. $75 \%$ de este material es carbón orgánico disuelto. Desde luego, la variación del nivel medio del mar, la amplitud de la marea, y los eventos de pluviosidad, pueden también incrementar la exportación de carbón orgánico desde los manglares.

El patrón de exportación de hojarasca y detritus orgánico particulado desde los manglares no está exclusivamente restringido a fuerzas geofísicas. En algunos casos se presentan importantes factores biológicos que influyen en la dinámica del detritus foliar, lo cual demuestra la importancia relativa de conocer los procesos geofísicos acoplados con la biodiversidad y su influencia sobre las funciones ecológicas del ecosistema manglar.

\section{Biodiversidad funcional}

Muchos organismos como los cangrejos desempeñan un papel importante en la estructura y flujo de energía de estos bosques a través de la dinámica del detritus de origen foliar. Aunque la alta diversidad de cangrejos y su efecto potencial en la

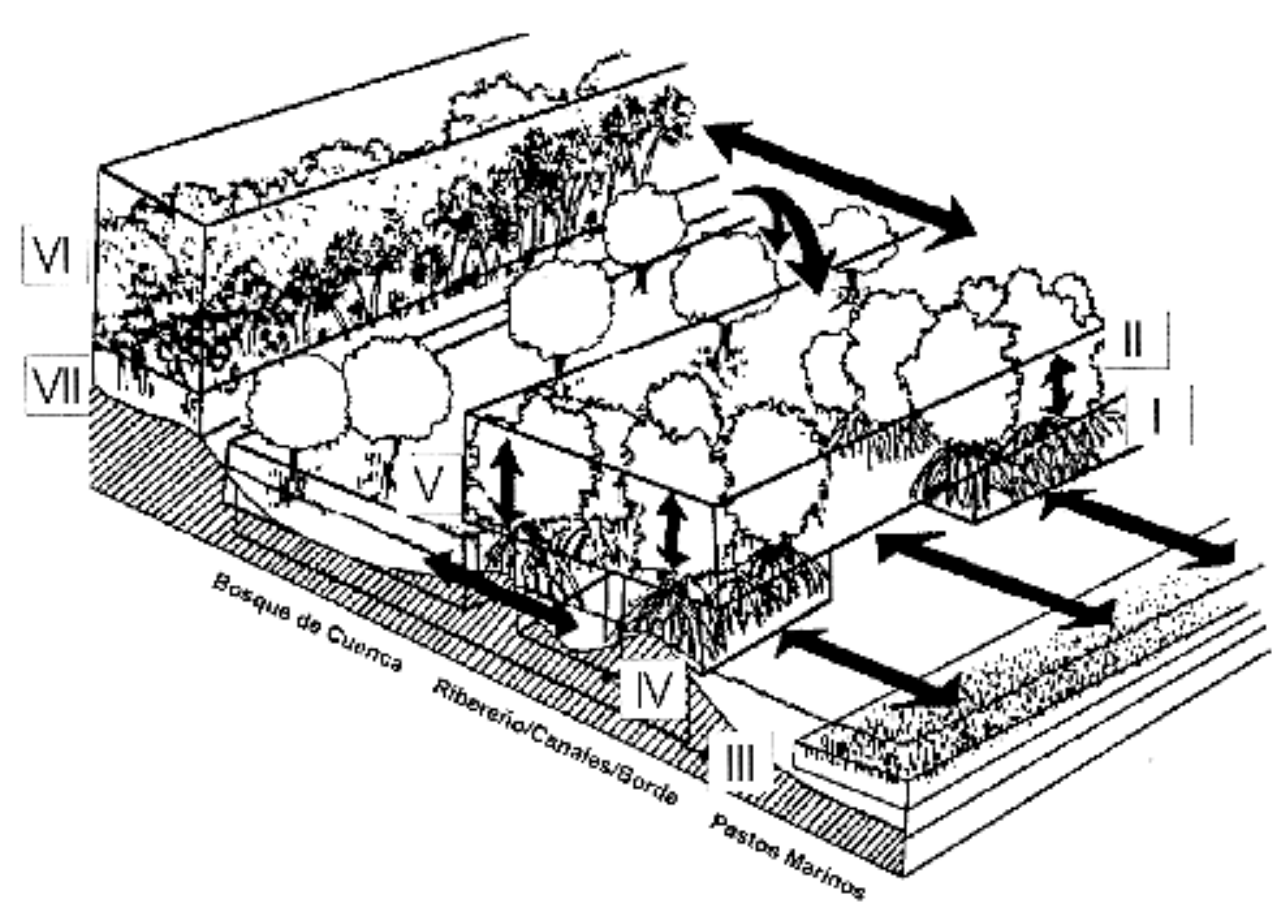

Figura 1. Tipos ecológicos de manglares y su distribución espacial dentro de la zona intermareal. Las flechas y los números romanos definen la ubicación y el movimiento de siete unidades específicas por asociación de hábitats principales en los tipos de manglares. I. Litoral/sublitoral, II. Follaje arbóreo, III. Comunidad bentónica e infauna, IV. Comunidad de canales de marea, V. Comunidad de la cuenca, VI. Comunidad arbórea tierra adentro, VII. Comunidad terrestre tierra adentro (según Twilley, Snedaker, Yáñez-Arancibia y Medina, 1996). 


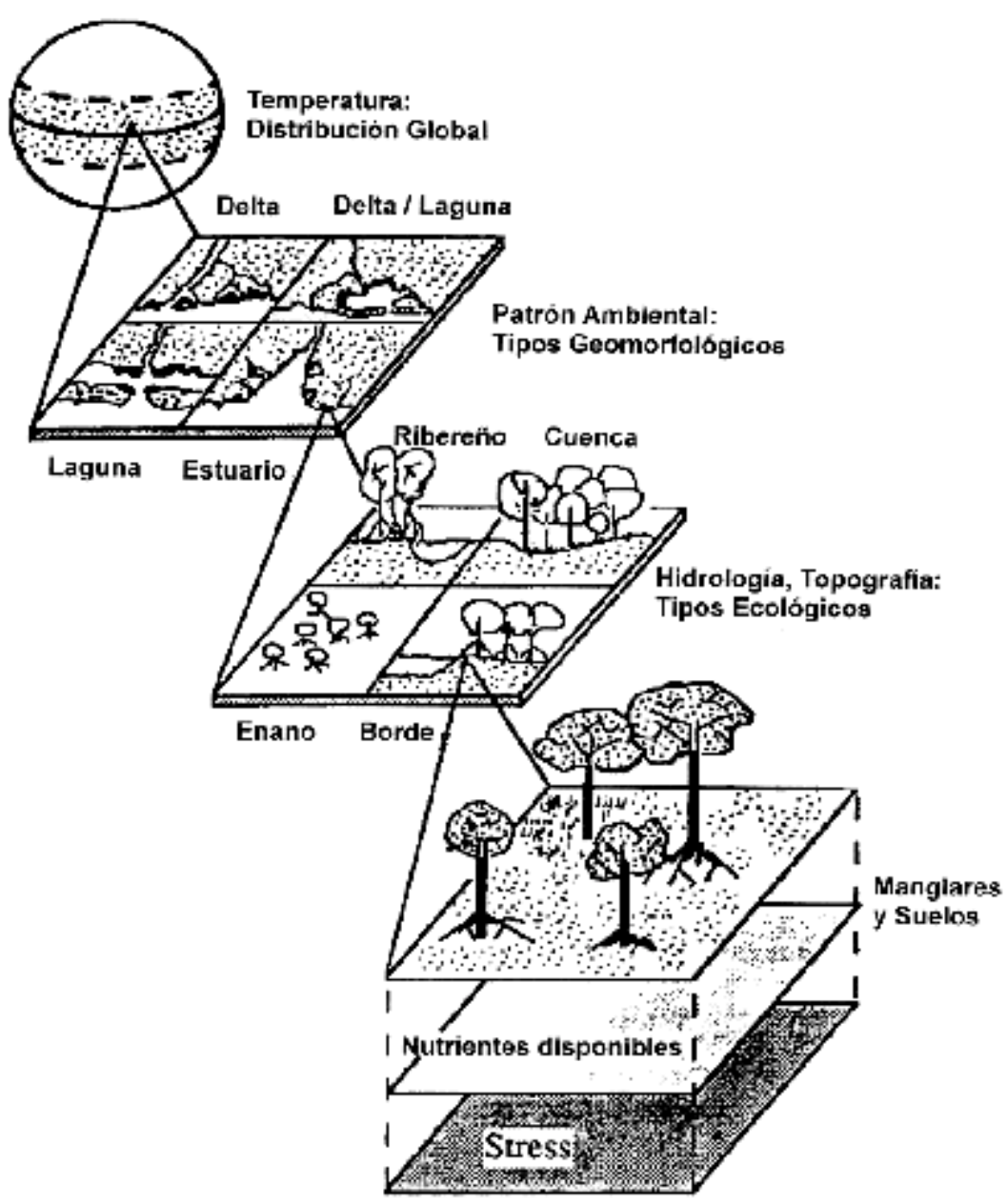

Figura 2. Sistema de clasificación jerárquica para describir diversos patrones de estructura y funcionamiento de los manglares, basado sobre factores que controlan la concentración de recursos de nutrientes y tensores en el suelo, p.e. global (temperatura), regional (geomorfológico), y local (ecológico). Según Twilley, Snedaker, Yáñez-Arancibia y Medina, 1996.

productividad del bosque de manglar ha sido bien reconocida (p.e. Uca, Sesarma, Chiromanthes, Cardisoma, Goniopsis, Ucides, Aratus), existe poca información cuantitativa sobre estructura de la comunidad, dinámica de las poblaciones, e interacciones ecológicas entre los cangrejos y la producción de detritus via hojarasca.

Existen evidencias que los cangrejos pueden remover por consumo entre el $9 \%$ y el $79 \%$ del detritus producido por hojarasca, dependiendo de la época del año, y del nivel de inundación, siendo esto uno de los principales vínculos entre productividad primaria y producción secundaria en ecosistemas de manglar. Más aún, se ha estimado que la tasa de renovación de hojarasca/detritus via cangrejos (ca. $14 \mathrm{~g} \mathrm{~m}^{2}$ día $^{-1}$ ), puede ser hasta 75 veces mayor que la tasa generada via microbiota.

Además, el detritus particulado por los cangrejos puede luego ser comido por ca. 50 especies de otros invertebrados y al menos 60 especies de peces. Por lo tanto, 


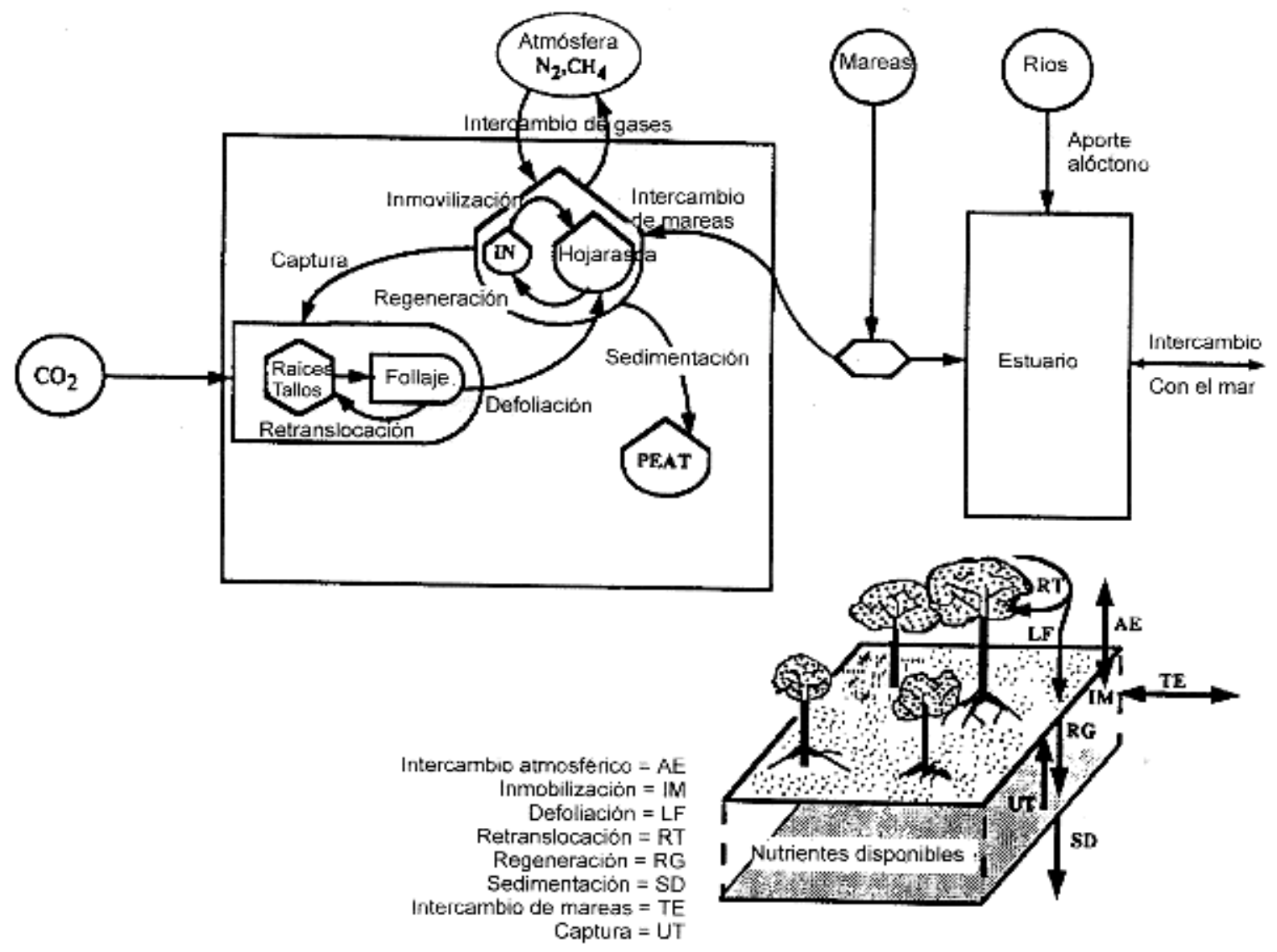

Figura 3. Flujos de materia orgánica y nutrientes en el ecosistema de manglar, incluyendo intercambio con el estuario (IN = nutrientes inorgánicos). Se presenta un diagrama del bosque de manglar con los recursos de nutrientes del sustrato, describiéndose el acoplamiento espacial en estos procesos ecológicos (según Twilley, Snedaker, YáñezArancibia y Medina, 1996).

el papel ecológico de estos macroinvertebrados es amplio ya que ellos: 1. Evitan que el material foliar de los manglares sea lavado fuera del bosque previo a su enriquecimiento, 2 . Proveen de materia orgánica particulada a los detritívoros que requieren de partículas finas como alimento, 3. Regulan el tamaño de la materia orgánica particulada en el ecosistema, 4. Estimulan la colonización de la materia orgánica particulada por la microfauna y microorganismos, permitiendo nutrientes disponibles para los árboles, 5. Simplifican la estructura y la composición química del detritus particulado, lo cual facilita la degradación por la microbiota, 6. Pueden afectar la estructura, composición de especies y expansión de los propágulos de los manglares, los cuales son comidos por los cangrejos entre un 75 y $100 \%$ de la producción total de epicotilos germinados.

Por otra parte, los peces estuarinos -como consumidores secundarios- en los ecosistemas de manglar, pueden ser muy importantes en el flujo de energía y materiales en diferentes maneras. Ellos pueden: 1. Almacenar nutrientes y energía, 2. Controlar el rango y magnitud 
del flujo de energía a través del consumo de fuentes alimentarias via pastoreo o detritus y, 3. Mover energía y nutrientes a través de las fronteras del ecosistema.

En términos generales, el nécton -organismos libre nadadores- utiliza los manglares como hábitat crítico para protegerse y alimentarse en las diferentes etapas de su ciclo de vida. La mayoría de los peces en estos ecosistemas son migratorios de pequeña escala, pero algunas especies pueden ser residentes permanentes. Principalmente se presentan 3 tipos de migración: 1. En ciclos diurnos, 2. En ciclos estacionales y, 3 . Ontogenéticos. Las migraciones diarias están en función de los hábitos alimentarios, mientras que las migraciones estacionales pueden estar relacionadas con parámetros ambientales como salinidad, temperatura, turbidez, o funciones biológicas como reproducción y reclutamiento; y todo esto en función de los pulsos de productividad primaria.

Los pulsos secuenciales de producción primaria por plancton y macrófitas como los manglares, acoplados con la exportación estacional de detritus, sugiere que la liberación de materia orgánica sostiene una alta producción secundaria y diversidad de especies consumidoras dependientes estuarinas. Las especies dominantes de peces actúan como controladoras de la estructura y función de los macro consumidores, mientras que la variabilidad físico-ambiental y productividad de los manglares modula su diversidad de especies. A través de la banda intertropical del planeta el número de especies de peces varía en un amplio rango que depende de las condiciones locales del manglar, la latitud, y la variabilidad ambiental, oscilando entre 20 y 200 especies.

La estructura funcional de los manglares y la diversidad de hábitats que sostiene, provee alimento y refugio a esta gran diversidad de peces a diferentes niveles tróficos. Esto se refleja claramente en la estructura trófica global del nécton y en los cambios en la dieta de las especies, de acuerdo con la época del año, la edad de los peces y la disponibilidad de alimento. Los peces relacionados a los manglares muestran lo siguiente: 1 . Flexibilidad de alimentación en tiempo y espacio, 2. Compartición de una fuente alimentaria común por un conjunto muy diverso de especies de peces, 3. Cada especie captura alimento desde diferentes niveles en la trama trófica, 4. La dieta cambia con el crecimiento, diversidad de alimento, y localidad dentro del estuario, 5 . Diversas especies utilizan tanto el patrón pelágico (pastoreo) como el bentónico (detritus) para la obtención de alimento. Estos elementos sugieren que la flexibilidad relativa de la estructura trófica de los macroconsumidores (peces y crustáceos) y su programación estacional para sincronizar la reproducción y la incorporación con los pulsos secuenciales de productividad primaria y aporte de materia orgánica, son mecanismos eficientes de acomodación frente a la dinámica físico-ambiental (p.e. los efectos en el litoral por el cambio climático global).

Por otra parte, la fragmentación de los paisajes del manglar crea el mismo problema -para los organismos migratorios- que el que se asocia con la fragmentación de bosques continentales. A su vez, la fragmentación del escenario manglar-pastos marinos 0 manglarcorales, reduce la complejidad del ecosistema y la diversidad nectónica, afectándose la estructura de la comunidad y las pesquerías locales.

\section{Valoración de las funciones ecológicas}

Los manglares se han degradado y manifiestan una evidente pérdida de su calidad ambiental. Esto se debe a diversas razones: 1. Cambios en la organización social de las comunidades humanas costeras, rotando la actividad económica entre pescador, campesino y artesano; 2. Incremento en el consumo de energía per cápita para desarrollar la economía 
costera; 3. Manejo fragmentado en el sector oficial, o ausencia total de un plan de manejo, ante la presión urbana, industrial, turística, agrícola y acuacultural; 4 . Depreciación del valor ecológico y uso irracional no sostenible; 5 . Poco impacto de los resultados científicos y baja disponibilidad de los mismos en términos prácticos para los usuarios del sector oficial; 6. Carencia de términos de referencia de vocación y aptitudes de la región y de evaluación ecológica y de recursos, para desarrollos productivos; y, 7. Reconversión de áreas de manglar para agricultura y acuacultura que colapsa en pocos años.

El valorar económicamente los ecosistemas de manglar ha avanzado con lentitud en México, puesto que estimar el valor económico de los servicios y funciones ecológicas implica cuantificar en términos monetarios la calidad del ambiente. Esencialmente, se deben incluir en esta valoración los usos y funciones ambientales clave que afectan actividades productivas locales y regionales, tales como el mantener la productividad de las pesquerías, contribuir a la estabilidad de la línea de costa, sostener la biodiversidad, establecer la relación con el turismo costero y determinar el uso potencial de los manglares como planta natural de tratamiento de aguas residuales.

Los manglares tienen valores que pueden caracterizarse por las siguientes funciones ecológicas: 1. Descarga y recarga de aguas subterráneas, 2 . Control de flujo y reflujo en el encuentro de las aguas dulces con las marinas, 3. Control de erosión y estabilización costera, 4. Retención de sedimentos, 5 . Retención de nutrientes, 6. Mantenimiento de la calidad del agua incluyendo transformación de nutrientes, 7. Estabilización microclimática, 8. Amortiguamiento de los contaminantes de ecosistemas vecinos, 9. Recreación y esparcimiento, 10. Incorporación biológica y 11. Hábitats de una alta biodiversidad.

La valoración económica de los manglares se justifica en términos de que la asignación actual de inversión o gasto público para la conservación de estos recursos, generalmente no refleja el valor que la sociedad les otorga. Asimismo, el establecimiento de actividades humanas en zonas costeras generalmente no incluye en sus estimaciones los valores de uso indirecto y de no-uso que pudieran ser impactadas por dicha actividad. Sin duda, es una necesidad para la gestión ambiental valorar económicamente los ecosistemas de manglar, $y$ obtener indicadores cuantitativos del daño económico por su degradación, ya sea por eventos naturales, acciones antrópicas, 0 el cambio climático global.

Estudios específicos han señalado que la valoración económica total de los manglares puede variar entre 38 dólares y 77 mil dólares por hectárea, dependiendo del método de valoración, el número de servicios ecológicos valorados, la latitud, el entorno social, la percepción económica, y la presión por el uso del suelo para reconvertir los hábitats.

\section{RESPUESTAS ESPERADAS DE LOS MANGLARES AL CAMBIO CLIMATICO GLOBAL EN LA ZONA COSTERA}

Los ecosistemas costeros,
incluyendo los manglares, son especialmente vulnerables debido a las presiones económicas y sociales, además de su localización sobre el borde costero (Figura 4). Alrededor del mundo, los manglares tienen un valor ambiental y ecológico, así como también proveen significativos beneficios socio-económicos a las economías de las comunidades tanto a nivel nacional como local. Estos ecosistemas, por su localización en la zona intermareal, se estima que serán de los ecosistemas mayormente afectados frente al cambio climático global, en particular frente a los efectos del incremento del nivel medio del mar, fuerza de vientos, oleaje y corrientes, y patrón de tormentas. 


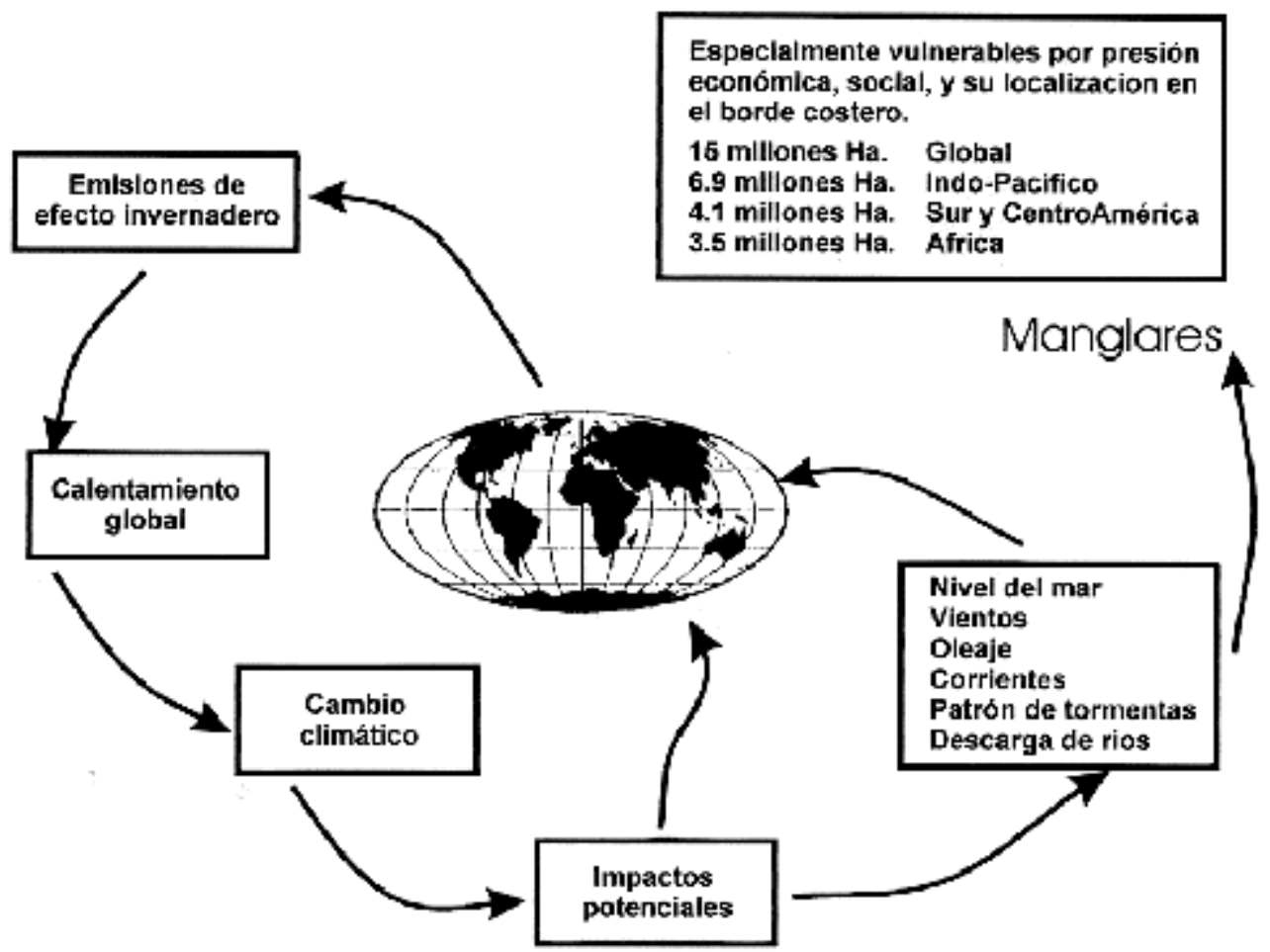

Figura 4. Secuencia de los efectos acoplados por el cambio global en la zona costera, p.ej. gases de efecto invernadero, calentamiento global, y cambio climático, induciendo impactos potenciales por incremento del nivel del mar, vientos, mareas, corrientes, patrón de tormentas y descarga de ríos. Los manglares son especialmente vulnerables debido a presiones sociales y económicas y su localización en la interfase tierra-mar.

\section{Respuestas esperadas del ecosistema al cambio climático global}

Como los manglares son uno de los más prominentes hábitats críticos costeros en latitudes tropicales y subtropicales alrededor del mundo, el impacto costero ante el cambio climático tendrá significado económico y social sobre este tipo de ecosistemas. Por sus características reproductivas, los manglares pueden acusar importantes respuestas sensitivas estructural y funcionalmente a los cambios climáticos de periodo largo. Otros ecosistemas tropicales y subtropicales, como otros humedales costeros salobres o dulceacuícolas y los pastos marinos, muestran una variabilidad más pronunciada en periodos cortos debido a fluctuaciones estacionales e interanuales y su tasa de renovación es muy rápida, por lo cual se dificulta su seguimiento frente al cambio climático global.

La Tabla 1 presenta resumidamente
los cambios esperados en la concentración de gases atmosféricos, en el promedio de temperatura global, y en el promedio del nivel del mar, según el Panel Intergubernamental sobre Cambio Climático (IPCC 1990). Se conoce que el calentamiento global puede estar causado por los gases del efecto-invernadero. Estos son gases que pueden absorber la radiación infrarroja. La absorción de radiación térmica de onda larga en la atmósfera evita el escape de la energía 
térmica, provocando el incremento marcado de la temperatura de la atmósfera. La Tabla 2 resume las principales respuestas esperadas de los ecosistemas costeros tropicales (manglares), frente a las predicciones sobre el cambio climático global.

\section{Efectos por el cambio climático global} Los ecosistemas tropicales estuarinos son también vulnerables a los cambios en el medio ambiente costero debido a las perturbaciones que resultan del incremento de gases de efecto invernadero en la atmósfera. Los manglares son un componente fundamental en los estuarios tropicales y se presentan en la interfase entre la tierra y el mar; por lo tanto, son muy sensitivos a los cambios en el nivel del mar. $\mathrm{El} \mathrm{CO}_{2} \mathrm{y}$ otros gases de efecto invernadero pueden llegar en el 2025 al doble de la concentración presente al comienzo de la revolución industrial, aumentando la temperatura superficial del planeta de 2 a $4{ }^{\circ} \mathrm{C}$ (Tabla 1).

Si la temperatura promedio se incrementa $3^{\circ} \mathrm{C}$ para el 2050 y permanece constante, el nivel del mar se incrementará ca. $1 \mathrm{~m}$ para el 2100 (50 a $91 \mathrm{~cm}$ por cada 100 años). Un calentamiento global de 6 ${ }^{\circ} \mathrm{C}$ para el 2100 podría resultar en un incremento del nivel del mar de $2.3 \mathrm{~m}$ (> $100 \mathrm{~cm}$ por cada 100 años según el Panel Intergubernamental sobre Cambio Climático) (IPCC 1990). Estos números son mayores que la tasa de incremento actual del nivel del mar, y son significativos en relación al ascenso del nivel del mar observado durante la última fase del Holoceno.

Existe mucha controversia sobre la tolerancia de los manglares al incremento del nivel del mar. Algunos sostienen que incrementos entre 12 y $27 \mathrm{~cm}$ por cada 100 años harán colapsar estos ecosistemas; además que descensos del nivel del mar producirían una mayor expansión de estos bosques. Sin embargo, hay evidencias que

los manglares no se verían afectados significativamente con ascensos del nivel del mar entre 50 y $80 \mathrm{~cm}$ por cada 100 años (p.e. Belice, Jamaica, Florida). Incluso durante los últimos 56 años, los manglares

Tabla 1. Condiciones globales inducidas por el desarrollo que se esperan para el próximo siglo

\begin{tabular}{||l|l|l|l||}
\hline GASES & DE & & CONCENTRACION \\
INVERNADERO & EN & CONCENTRACION \\
LA ATMOSFERA & EN 1990 & $\begin{array}{l}\text { TASA DE CAMBIO } \\
\text { ACTUAL (ANUAL) }\end{array}$ & $\begin{array}{l}\text { ESPERADA } \\
\text { PARA EL 2100 }\end{array}$ \\
\hline CO $^{2}$ & $353 \mathrm{ppmv}$ & $840 \mathrm{ppmv}$ \\
Metano & $1.72 \mathrm{ppmv}$ & $4 \mathrm{ppmv}$ \\
CFC-11 & $280 \mathrm{pptv}$ & $630 \mathrm{pptv}$ \\
CFC-12 & $480 \mathrm{pptv}$ & & $1400 \mathrm{pptv}$ \\
Oxido Nitroso & $310 \mathrm{ppbv}$ & 0,0965 & $420 \mathrm{ppbv}$ \\
\hline
\end{tabular}

Temperatura global media $+0.3^{\circ} \mathrm{C}$ por década; incertidumbre 0.2 a $0.5^{\circ} \mathrm{C}$ por década; incremento $+3.0^{\circ} \mathrm{C}$ al año 2100.

La superficie continental se calentará más rápidamente que los océanos; expansión térmica y deshielo.

Nivel medio global del mar $+6.0 \mathrm{~cm}$ por década; incertidumbre 3.0 a $10 \mathrm{~cm}$ por década; incremento $+60.0 \mathrm{~cm}$ al año 2100.

Lluvias sin patrón global fijo; incremento de lluvias torrenciales y disminución de lluvias ligeras; incremento de frecuencia e intensidad de huracanes y ciclones. Mayor intensidad del fenómeno "El Niño".

Fuentes: IPCC, 1990. Climate change: The scientific assessment. Report Working Group 1. Cambridge University Press. Cambridge UK, y, UNEP, 1994. Assessment and monitoring of climatic change impacts on mangrove ecosystems. UNEP Regional Seas Reports and Studies No. 154. 
Tabla 2. Principales respuestas esperadas de los escosistemas costeros tropicales frente a las predicciones sobre los cambios climáticos

INCREMENTO DEL NIVEL MEDIO DEL MAR

La comunidad de manglar progradará tierra adentro si hay suficiente ambiente sedimentario y sin obstáculos topográficos.

La erosión sobre el margen litoral se incrementará.

El rango del nivel medio del mar determinará el nivel de reestablecimiento de los manglares, dunas y humedales costeros.

La productividad secundaria (y primaria acuática) se incrementará por la mayor disponibilidad de nutrientes debido a la erosión y resuspensión.

\section{INCREMENTO EN LA CONCENTRACION DE CO² ATMOSFERICO}

La fotosíntesis del follaje de los manglares no se incrementará significativamente.

La eficiencia de los manglares en el uso del agua se mejorará. Esto puede o no verse reflejado en el crecimiento.

No todas las especies de manglares responderán de igual manera.

INCREMENTO EN LA TEMPERATURA $\left({ }^{\circ} \mathrm{C}\right)$ ATMOSFERICA

Algunas poblaciones de manglares extenderán su distribución hacia latitudes mayores.

En muchas especies de manglares habrá cambios en los patrones fenológicos, reproductivos y de crecimiento.

Se incrementará la productividad neta global del ecosistema de manglar.

La biodiversidad de plantas y animales en los manglares se incrementará (beneficios de microclima) y cambiará la composición florística y faunística.

Se acelerarán los procesos microbianos en los manglares, en la interfase agua-sedimento

CAMBIOS EN EL PATRON DE LLUVIAS

Los cambios en el contenido de agua del suelo y salinidad del sustrato, tendrán significativo impacto sobre el crecimiento de los manglares.

Un incremento de la precipitación sobre la tasa de evapotranspiración incrementará la tasa de producción primaria de los manglares.

Un incremento en la salinidad del suelo reducirá la productividad primaria y crecimiento de los manglares.

La fauna eurihalina no se verá afectada por el incremento en salinidad pero la distribución de especies estenohalinas se alterará significativamente.

IMPACTO ESPERADO DE LOS CAMBIOS CLIMATICOS SOBRE EL USO DEL SUELO, UTILIZACION Y EXPLOTACION DE LOS MANGLARES

Se incrementará el riesgo de inundación de tierras bajas.

Se incrementará la erosión de los litorales blandos vulnerables.

Se incrementará el riesgo de intrusión salina.

Se incrementará la frecuencia del daño causado por tormentas.

Fuente: UNEP, 1994 Assessment and monitoring of climatic change impacts on mangrove ecosystems. UNEP Regional Seas Report and Studies No. 154. 
de Key West Florida se han expandido hacia el mar y hacia el continente, a pesar de los huracanes y aún con un incremento del nivel del mar equivalente a $23 \mathrm{~cm}$ por cada 100 años.

Los cambios en la riqueza de especies de los manglares durante la migración horizontal hacia el continente en respuesta a los cambios del nivel del mar, dependen de las respuestas específicas de cada especie del manglar al incremento en la inundación y erosión, y a los efectos del tamaño del propágulo y ritmo de la marea a lo largo de la zona intermareal.

Estos dos factores indican que la profundidad de la inundación mareal puede ser el principal factor en regular la zonación de especies con el ascenso del nivel del mar. La mayoría de los estudios señalan que Rhizophora es más tolerante a la baja disponibilidad de oxígeno causada por la inundación mareal y la acumulación de agua que Avicennia. Si no hay barreras geográficas continentales, los manglares pueden migrar tierra adentro manteniendo a prudente distancia el incremento del nivel del mar. Asumiendo como constantes otros factores ecológicos, Rhizophora con propágulos de gran tamaño y mayor tolerancia a las inundaciones invadiría y dominaría las zonas altas previamente ocupadas por Avicennia y Laguncularia, las cuales se retraerían hacia la nueva zona salina somera intermareal formada hacia el interior. Los manglares de borde compuestos básicamente por Rhizophora irían desapareciendo paulatinamente en correspondencia con su nueva distribución hacia el interior.

La temperatura es el factor climático básico que modula los límites de distribución de los manglares hacia el norte y hacia el sur del planeta. Se ha señalado que los bosques de manglares responderían a la disminución de la temperatura reduciendo la riqueza de especies, simplificando la estructura del bosque (altura y biomasa). Aunque el promedio de temperatura del aire y del agua muestran alguna correlación con la distribución de los manglares en el mundo, las temperaturas extremas pueden ser el principal factor de control. Avicennia y Laguncularia parecen ser más tolerantes a las temperaturas frías en el neotrópico que Rhizophora. Las diversas tolerancias a las bajas temperaturas entre diferentes especies de mangles, generalmente se infiere a su distribución natural y adaptaciones morfo-fisiológicas, metabólicas y reproductivas. Sin embargo, se ha demostrado que la diversidad genética influye en la tolerancia de los manglares al cambio global.

Diversos estudios indican que la frecuencia $e$ intensidad de tormentas tropicales y huracanes se están incrementando bajo condiciones de calentamiento climático. Puesto que los manglares están distribuidos en latitudes donde la frecuencia de huracanes es alta, es importante comprender cómo estos eventos afectan el desarrollo de los bosques (p.e. estructura del bosque, composición de especies) y la dinámica de la comunidad del ecosistema de manglar, incluyendo biodiversidad. Aún cuando el efecto es evidente en la defoliación, producción de hojarasca y la mortalidad de los árboles, también hay evidencias de una rápida recuperación, por este daño, aunado al papel que juega la dinámica del suelo controlando el crecimiento y desarrollo de los árboles.

\section{CONCLUSIONES}

El cambio climático global está afectando la dinámica natural de la biósfera. La zona costera es una ecoregión sensible donde los cambios inducidos y sus efectos, se están apreciando en tiempo real, con mayor celeridad que lo previsto. Los manglares, ubicados en la interfase tierra-mar controlan interacciones entre hábitats críticos de los humedales costeros con el océano adyacente. No obstante, son 
ecosistemas frágiles que están acusando el impacto frente al cambio climático, pero existen evidencias que estos humedales forestados tienen gran capacidad de acomodación para contender con mejor éxito que otros sistemas naturales, frente a esta variabilidad físico ambiental de ritmo acelerado.

La respuesta final de los ecosistemas de manglar estará determinada por el balance dinámico entre la tasa de incremento del nivel del mar, la acreción sedimentaria y la migración lateral. Cuando el incremento del nivel del mar es mucho mayor que la acreción sedimentaria y el traslado de la línea de costa sobrepasa la tasa a la cual pueden migrar los manglares, el ecosistema se sumergirá y será reemplazado por un ambiente costero marino. Si la tasa del incremento del nivel del mar es mayor que la acreción sedimentaria, pero la tasa de traslado de la línea de costa no sobrepasa la capacidad del ecosistema de migrar tierra adentro, el bosque podrá retraerse tierra adentro. Finalmente si el incremento del nivel del mar es relativamente pequeño, los manglares pueden permanecer en la línea de costa actual acumulando sedimentos en la vertical del sustrato. El ensayo predictivo de esta aproximación conceptual puede variar significativamente cuando otros factores, como el $\mathrm{CO}_{2}$ y la temperatura, son incorporados en los modelos matemáticos que actualmente se diseñan para estimar respuestas al cambio climático global.

Estudios existentes sugieren que la dominancia alterna entre Avicennia y Rhizophora de acuerdo con gradientes de zonación, balancean la dinámica del nitrógeno entre el follaje y el suelo del ecosistema de manglar, con la ventaja de ser un mecanismo flexible frente al cambio climático global y su efecto en los litorales tropicales.

Asimismo, otros estudios sugieren que la flexibilidad relativa de la estructura trófica de los macro-consumidores (peces y crustáceos) y su programación estacional para sincronizar la reproducción y la incorporación con los pulsos secuenciales de productividad primaria y aporte de materia orgánica, son mecanismos eficientes de acomodación frente a los efectos físico ambientales del litoral por el cambio climático global.

Dado que, de manera general, los manglares son ecosistemas subvalorados económicamente, es una necesidad para la gestión ambiental el valorarlos con precisión y el obtener indicadores cuantitativos del daño económico ocasionado por su degradación, ya sea por eventos naturales, acciones antrópicas, o el cambio climático global.

Existe controversia sobre la tolerancia de los manglares al incremento de gases de efecto invernadero, de temperatura, de ascenso del nivel del mar y de frecuencia e intensidad de tormentas tropicales y huracanes, más aún cuando esta variabilidad se está presentando a un ritmo mayor que el inicialmente predicho. Sin embargo, la estructura funcional de este sistema ecológico, así como las evidencias revisadas permiten plantear la hipótesis que "los manglares como hábitat forestado crítico de la zona costera presentan respuestas de acomodación frente a la variabilidad ambiental que induce el cambio global, desarrollando un papel estructural $y$ funcional clave en la estabilidad de la línea de costa, la persistencia de hábitats y biodiversidad, el metabolismo del ecosistema, reduciendo riesgos e incertidumbre para el desarrollo sustentable del uso de sus recursos".

\section{RECONOCIMIENTOS}

La tercera autora desea expresar su reconocimiento al CONACYT por el apoyo otorgado a través de la beca 119112 / 120940 / 1998-2001. 


\section{BIBLIOGRAFIA}

Alusa, A.L. y L.J. Ogallo. 1992. Implications of expected climate change in the Eastern African coastal region: An overview. UNEP Regional Seas Report and Studies 149. 28 pp.

Bacon, P.R. 1994. Template for evaluation of impacts of sea level rise on Caribbean coastal wetlands. Ecol. Eng. 3: 171-186.

Boto, K.G. 1982. Nutrient and organic fluxes in mangroves. In: B.F. Clough (Ed.) Mangrove Ecosystem in Australia: Structure, Function and Management. Australian National University Press, Camberra. p: 239257.

Dixon, J.A. 1989. Valuation of mangroves. Tropical Coastal Area Management 4: 1-11. ICLARM. Manila.

Downing, J., N. Rabalais, J. Elser, J.M. Melak. R.R. Twilley, M. McClain, W.M. Lewis, J. Corredor, G. Turner, D. Soto, A. Yáñez-Arancibia y R.W. Howarth. 1998. The impact of land-use-changes on the global $\mathrm{N}$-cycles in tropical aquatic ecosystems: Current conditions and projected changes. 40pp. Biogeochemistry (en prensa).

Eamus, D. y P.G. Jarvis 1989. Direct effects of increase in the global atmospheric $\mathrm{CO}_{2}$ concentration on natural and commercial temperate trees and forests. Advances in Ecological Research 19: 1-55.

Ellison, J.C. 1993. Mangrove retreat with rising sea level in Bermuda. Estuarine Coastal Shelf Science 37: 75-87.

Ellison, J.C. y D.R. Stoddart. 1991. Mangrove ecosystem collapse during predicted sea-level rise: Holocene analogues and implications. Journal of Coastal Research 7:151-165.
Gilmore, R.G. y S.C Snedaker. 1993. Mangrove forest. In: Martin, W.H., S. Boyce y K. Echternacht (Eds.) Biodiversity of the Southeastern United States: Lowland Terrestrial Communities. Wiley. New York. p: 165-198.

IOC. 1991. UNEP-IOC-WMO Meeting of Experts on Long-Term Global Monitoring System of Coastal and Nearshore Phenomena Related to Climate Change. Report of IOC Experts and Equivalent Bodies. IOCUNESCO. París. 30 p.

IPCC. 1990. Potential impacts of climate change. Report of Working Group II to Intergovernmental Panel on Climate Change. World Meteorological Organization. Genova.

IPCC. 1990. Climate change: The scientific assessment. Report Working Group 1. Cambridge University Press. Cambridge, UK.

Kjerfve, B. y L.D. Lacerda. 1991.Variacao relativa do nivel do mar. Ciencia Hoje (14) $81: 60-65$.

Kjerfve, B., W.K. Michener y L.R. Gardner. 1991. Impacts of climate change in estuary and delta environments. Symposium on Impacts of Climte Change on Ecosystems and Species. The Netherlands 2-6 Dec. 1991. UICN. $18 \mathrm{p}$.

Lara-Domínguez, A.L., A. Yáñez-Arancibia y J.C. Seijo. 1998. Valuación económica de los servicios de los ecosistemas: Estudio de caso de los manglares en Campeche. In: CONABIO-INE (Ed.) Aspectos Económicos sobre la Biodiversidad de México. CONABIO. INE. SMARNAP. México, D.F. p: 23-44.

Lugo, A.E., S. Brown y M.M. Brinson. 1990. Concepts in wetland ecology. In: Lugo, A.E., M.M. Brinson y S. Brown 
(Eds.) Ecosystems of the World 15. Forested Wetlands. Elsevier, Amsterdam p: 53-85.

Milliman, J.D., J.M. Broadus y F. Gable. 1989. Environmental and economic implications of rising sea level and subsiding deltas: The Nile and Bengal examples. Ambio 18: 340-345.

National Research Council. 1990. Sealevel change. National Academy of Science Press. Washington, D.C. 234 p.

Odum, W.E., C.C. Mclvor y T.J. Smith. 1982. The Ecology of Mangrove of South Florida: A Community Profile. Fish and Wildlife Service/Office of Biological Services. Washington, D.C. FWS/OBS-81/24.144 p.

Ogden, J.C. y E.H. Gladfelter. 1983. Coral reefs, seagrass beds and mangroves: Their interactions in the coastal zones of the Caribbean. UNESCO Studies in Marine Sciences 40. 96 p.

Parkinson, R.W. 1992. Building a conceptual model of mangrove ecosystems response to global climate change: Sea-level rise and the geologic record. Jaina EPOMEX Newsletter 3 (3): 6.

Peltier, W.R. y A.M. Tushingham. 1989. Global sea-level rise and the greenhouse effect: Might they be connected?. Science 244: 806-810.

Robertson, A.I. y D.M. Alongi (Eds.). 1992. Tropical Mangrove Ecosystems. Coastal and Estuarine Studies 41. American Geophysical Union. Washington D.C. 329 p.

Rojas Galaviz, J.L., A. Yáñez-Arancibia, J.W. Day y F. Vera Herrera. 1992. Estuarine primary producers: Laguna de Terminos a case study. In: U. Seeliger (Ed.) Coastal Plant
Communities of Latin America. Academic Press Inc. New York. Cap. 10: 141-154.

Saenger, P., E.J. Hegerl y J.D.S. Davie. 1983. Global Status of Mangrove Ecosystems. Commission of Ecology Paper No. 3. IUCN. Gland, Switzerland $88 \mathrm{p}$.

SEMARNAP. 1997. México: Primera Comunicación Nacional para la Convención Marco de las Naciones Unidas ante el Cambio Climático. Semarnap. México, D.F. 149 p.

Smith, T.J., K.G. Boto, S.D. Frusler y R.L. Geddins. 1991. Keystone species and mangrove forest dynamics: The influence of burrounding by crabs on soil nutrient status and forest productivity. Estuarine and Coastal Shelf Sci. 33: 419-432.

Smith, T.J., M.B. Robblee, H.R. Wanless y T.W. Doyle. 1994. Mangroves, hurricanes, and ligthning strikes. BioScience 44: 256-262.

Snedaker, S.C. 1993. Impact on mangrove. In: G.A. Maul (Ed.) Climate Change in the Intra-Americas Sea. Edward Arnold, Kent. p: 282-305.

Snedaker, S.C. y C.D. Sylva. 1987. Impact of climatic change on coastal resources: Implications for property values, commerce, estuarine environments and fisheries, with special reference to South Florida. Symposium Climatic Change in South United States. New Orleans, LA. 61 pp.

Snedaker, S.C., J.F. Meeder, M.S. Ross y R.G. Ford. 1994. Discussion of J.C. Ellison and D.R. Stoddart, Mangrove ecosystem collapse during predicted sea-level rise: Holocene analogues and implications. J. Coastal Res. 7: 
151-165, 1991. J. Coastal Res.10:497-498.

Stewart, R.W, B. Kjerfve, J. Milliman y S.N. Dwivedi. 1990. Relative sea-level change: A critical evaluation. UNESCO Report in Marine Science 54: 1-22.

Sylva, D. 1986. Increased storms and estuarine salinity and other ecological impacts of the greenhouse effect $I n$ : Titus, J.G. (Ed.) Effects of Changes in Stratospheric Ozone and Global Climate. Volume 4: Sea-level Rise. US-EPA. p:153-164.

Twilley, R.R. 1988. Coupling of mangroves to the productivity of estuarine and coastal waters, In: B.O. Jansson (Ed.) Coastal-offshore Ecosystem Interactions. Springer Verlag, Berlin. p:155-180.

Twilley, R.R. 1995. Perspectives on mangrove ecosystems related to the energy signature of coastal environments. In: C.H. Hall (Ed.) Maximum Power. University of Columbia Press. Niwot, Colorado. p: 43-62.

Twilley, R.R., R.H. Chen y T. Hargis. 1992. Carbon sinks in mangroves and their implications to carbon budget to tropical coastal ecosystems. Water. Air Soil Pollution. 64: 265-288.

Twilley, R.R., S.C. Snedaker, A. YáñezArancibia y E. Medina. 1996. Biodiversity and ecosystem processes in tropical estuaries: Perspectives on mangrove ecosystems. In: H.A. Mooney, S.H. Cusham, E. Medina, O.E. Sala y E.D. Schultze (Eds.) Functional Roles of Biodiversity: A Global Perspective. John Wiley \& Sons. Ltd. Cap. 13: 327-370.
UNEP. 1994. Assessment and monitoring of climatic change impacts on mangroveecosystems. UNEP Regional Seas Report and Studies No. 154.62 p.

Woodroffe, C.D. 1990. The impact of sealevel rise on mangrove shoreline. Progress in Physical Geography 14: 483-520.

Yáñez-Arancibia, A., G. Soberón-Chávez y P. Sánchez-Gil. 1985. Ecology of control mechanisms of natural fish production in the coastal zone. In: A. Yáñez-Arancibia (Ed.) Fish Community Ecology in Estuaries and Coastal Lagoons: Towards an Ecosystem Integration. Cap. 27: 571594. UNAM Press. Mexico, D.F. 654 p.

Yáñez-Arancibia, A., A.L. Lara-Domínguez y J.W. Day. 1993. Interactions between mangrove and seagrass habitats mediated by estuarine nekton assemblages; Coupling of primary and secondary production. Hidrobiología 264: 1-12.

Yáñez-Arancibia, A. y A.L. LaraDomínguez. 1995. Los manglares de América Latina en la encrucijada. Faro ICSED Santiago de Chile y Univ. Rhode Island. 1 (1): 1-7.

Yáñez-Arancibia, A. y A.L. LaraDomínguez (Ed.). 1999. Ecosistemas de Manglar en América Tropical. Instituto de Ecología A.C. Xalapa, México. UICN Mesoamérica Costa Rica, NOAA-NMFS. Beoufort, NC. EUA. 350 p.

Yáñez-Arancibia, A., P. Sánchez-Gil y A.L. Lara-Domínguez. 1999. Ecological functional groups, seasonality, and biodiversity in Términos Lagoon a tropical estuary, Mexico. Revista Mex. Hist. Nat. Vol. 49 (en prensa). 